\title{
DYNAMICS OF PLANE STELLAR SYSTEMS
}

FRANK HOHL

NASA, Langley Research Center, Hampton, Virginia

\begin{abstract}
The evolution of initially balanced rotating disks of stars is investigated with a computer model for isolated disks of stars. An isolated, initially cold balanced disk is found to be violently unstable. Adding a sufficient amount of velocity dispersion will stabilize all small-scale disturbances. However, the disks are still unstable against slowly growing long wave-length modes and after about two rotations most disks tend to assume a bar-shaped structure. It is found that the final mass distribution over most of the disk can be closely approximated by an exponential variation, irrespective of the initial mass distribution. The gravitational two-stream instability is investigated by means of a modified computer model for infinite doubly periodic stellar systems.
\end{abstract}

\section{Introduction}

The only means available to us for performing gravitational experiments on the evolution of stellar systems is the use of computer models. Such numerical methods can be called 'experimental stellar dynamics' and they play a role similar to the experimental methods in physics. The type of computer model used in the present paper applies to systems that can be considered collisionless. The force acting on a particular star is determined by the mean gravitational field of the system and the effect of nearby stars can be neglected. Chandrasekhar (1960) points out that for the Galaxy a star can describe at least 100 rotations about the galactic center before the effects of encounters with other nearby stars becomes important. A galaxy can therefore be studied by using a 'collisionless' computer model in which close encounters are effectively neglected. The first such models used were simple one-dimensional models, where the stars are stratified into plane parallel layers or mass sheets (Lecar, 1966; Hohl and Feix, 1967; Hohl, 1968a). The one-dimensional model is useful as an approximation to the motion of stars normal to the galactic plane of the Galaxy. The one-dimensional model was also used to investigate 'violent relaxation' of selfgravitating systems (Hohl and Campbell, 1968, 1969; Cuperman et al., 1969). Henon (1964) used a model of concentric spherical mass shells to study the dynamical mixing of spherical star clusters. A model of concentric mass rings was used by Toomre (1954). Two-dimensional models, in which the motion of infinitely long mass rods is followed, have been used by Hockney (1967) and by Hohl (1968b, 1969).

Two computer models for self-consistent disk galaxies have recently been described (Miller and Prendergast, 1968; Hohl and Hockney, 1969). The model of Miller and Prendergast is doubly periodic and the forces, star positions and velocities are allowed to attain only discrete (integer) values which are less than some given maximum value. In a recent report Miller et al. (1970) used their model to study the evolution of spiral structure and they find that spiral patterns persist for about three galactic rotations. This spiral pattern was obtained for the 'gas' component of systems which consisted of 'gas' and stars. The model described by Hohl and Hockney is 
for isolated disk galaxies and the star positions and velocities are allowed to attain essentially any value. Hockney and Hohl (1969) used the model to study the effects of velocity dispersion on the stability of an initially balanced uniformly rotating disk of stars. To investigate the development of spiral structure the model was modified by Hohl $(1970 \mathrm{a}, \mathrm{b})$ to include a fixed central force similar to the Schmidt model of the Galaxy. It was then found that spiral structure persisted for more than eight rotations.

In the present paper the evolution and final state of self-consistent and initially stationary disks of stars is investigated by means of the computer model for isolated disks of stars developed by Hohl and Hockney (1969). A number of initially cold (zero velocity dispersion) and balanced disk galaxies were investigated previously (Hohl, 1970) and were found to be violently unstable. The results presented here are for disks with an initial velocity dispersion equal to that required for stabilization of all axisymmetric instabilities as calculated by Toomre (1964). Such an initial velocity dispersion will stabilize all small-scale disturbances but the disk is still unstable against slowly growing nonaxisymmetric disturbances which cause the disk to assume a bar-shaped structure in less than two rotations. The development of a bar-like structure appears to be a general feature for disks of stars and has been found to occur for a large variety of initial conditions (Hohl, 1970). The later stages of the calculations by Miller et al. (1970) also give an indication of the development of a bar-like structure.

\section{The Model}

The model used for the present calculations is described in detail by Hohl and Hockney (1969) and a listing of the computer program used for the potential calculation is given elsewhere (Hohl, 1970). We include here only a summary of the model.

The model for the disk galaxy consists of a large number of representative stars (here 50000 or 100000 ) that are confined to move in the galactic disk. An $N$ by $N$ (here 64 by 64 or 128 by 128) array of cells is superposed over the plane of the disk for the purpose of calculating the gravitational potential. At the center of each cell a mass density is defined which is given by the number of stars in that cell. The mass density distribution is used to obtain the gravitational potential at the center of each cell. From the gravitational potential the force acting at the position of a star is calculated by means of a bilinear interpolation among four surrounding cell centers. Newton's equations of motion are then used to advance the position and velocity of each star by a small time step. Usually there are 200 or 400 time steps per 'galactic rotation'. One complete cycle for advancing the motion of the system by a small time interval consists of the following steps:

(i) The coordinates of each star are examined to determine the mass density over the $N$ by $N$ array of cells.

(ii) The gravitational potential corresponding to the mass density distribution is determined by using fast Fourier transform methods.

(iii) The gravitational potential is used to advance the motion of all the stars by 
the use of time centered finite difference equations. The cycle repeats at step (i). If a star leaves the $N$ by $N$ array of cells it is still included in the calculations by approximating the force acting on the star. For the doubly periodic system a star leaving the array at one side will enter again at the opposite side with the same velocity components.

The effects of varying the number of stars and various other discretization parameters was investigated by Hohl and Hockney (1969). For a 50000 star system the effect of binary collisions for the model has been estimated (Hohl, 1970) to be such that the collision time equals about 100 'galactic rotations'.

\section{Gaussian Mass Distribution}

\section{A. INITIAL CONDITIONS}

The first system to be investigated is a disk with a Gaussian surface mass density distribution given by

$$
\mu(r)=\mu(0) \exp \left\{-\pi \frac{\mu(0)}{M} r^{2}\right\}
$$

where $M$ is the total mass of the disk. For the present calculation the value of $1 /(\pi \mu(0) / M)$ was taken to be $50 \mathrm{kpc}^{2}$. The rotational velocity required to balance a cold disk with a Gaussian surface density was calculated by Toomre (1963) as

$$
V_{\theta}^{2}=\pi^{2} G \mu(0) \sqrt{\frac{\sigma(0)}{M}}{ }_{1} F_{1}\left(1.5 ; 2 ; \frac{\pi \mu(0)}{M} r^{2}\right)
$$

where ${ }_{1} F_{1}(1.5 ; 2 ; x)$ is the confluent hypergeometric function. Toomre (1964) found the root-mean-square radial velocity dispersion required to stabilize all axisymmetric disturbances anywhere in the disk to be

$$
\sigma_{r, \min }=3.36 \mathrm{G} \mu / \kappa
$$

where $\sigma$ and $\kappa$ are the local values of the density and epicyclic frequency respectively. To determine whether Toomre's minimum velocity dispersion will stabilize a disk with a Gaussian mass distribution the initial velocity dispersion is taken as

$$
\sigma_{r}(r)=\sigma_{r, \min }
$$

for the radial component and

$$
\sigma_{\theta}(r)=\frac{\kappa(r)}{2 \omega_{0}(r)} \sigma_{r, \min }
$$

for the azimuthal component. The angular velocity $\omega_{0}(r)$ is defined by

$$
\omega_{0}^{2}=\frac{1}{r} \frac{\partial \phi}{\partial r}
$$

where $\phi$ is the gravitational potential. Because of the effective pressure caused by 
the added velocity dispersion the initial angular velocity of the stars, $\omega(r)$, for a balanced disk is lower than $\omega_{0}(r)$ and is given by

$$
\omega^{2}=\omega_{0}^{2}+\frac{1}{r \mu(r)} \frac{\partial}{\partial r}\left(\mu(r) \sigma_{r}^{2}(r)\right)+\frac{1}{r^{2}}\left[\sigma_{r}^{2}(r)-\sigma_{\theta}^{2}(r)\right] .
$$

Figure 1 shows the variation of $\omega_{0}, \omega$ and $\kappa$ as a function of $r$ for the disk with a Gaussian mass distribution.

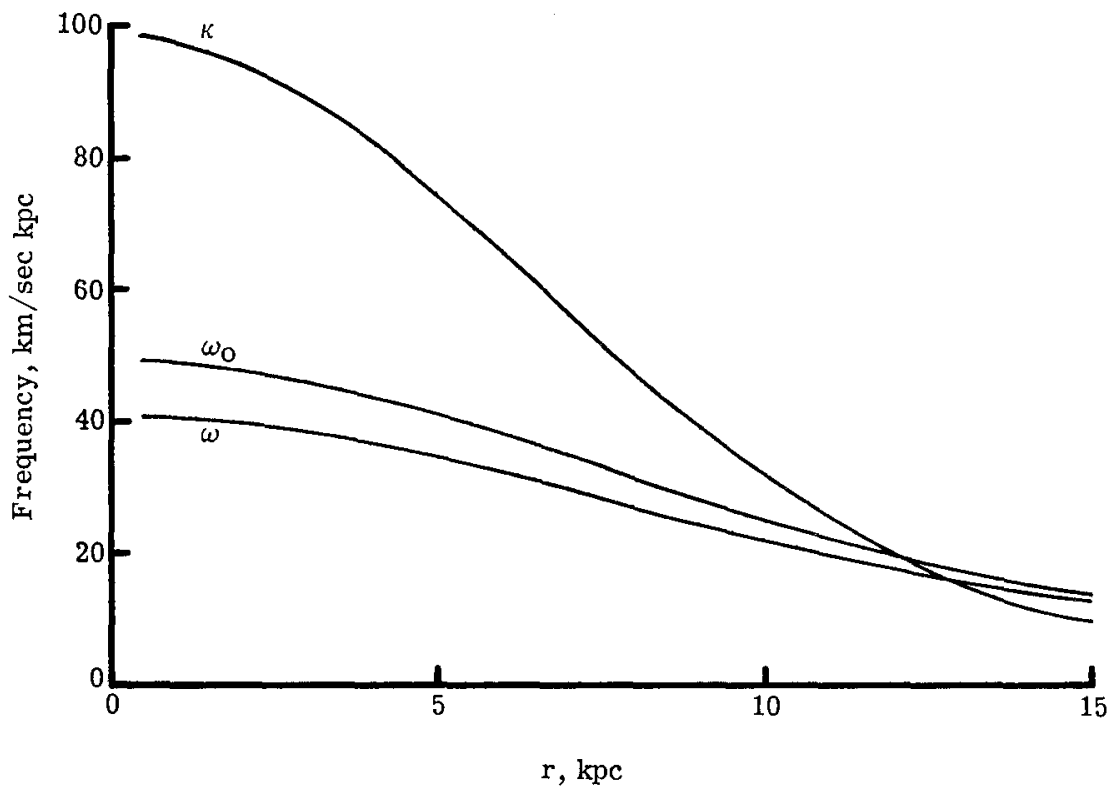

Fig. 1. Variation of $\omega_{0}, \omega$, and $\kappa$ as a function of $r$ for a disk of stars with a Gaussian mass density. The angular velocity $\omega_{0}$ is that for a cold balanced disk, $\omega$ is given by Equation (6), and $\kappa$ is the epicyclic frequency.

\section{B. EVOLUTION OF THE DISK}

The evolution of the disk is shown in Figure 2. There are 50000 stars each of mass $G m=77.4$ in the disk and 200 time steps per rotation are used in the calculation. The time is given in units of the rotational period of the cold balanced disk

$$
\tau_{0}=2 \pi / \omega_{0}(r)
$$

at $r=10 \mathrm{kpc}$. The rectangular border enclosing the disk is at $x= \pm 19 \mathrm{kpc}$ and $y=$ $\pm 19 \mathrm{kpc}$. The evolution displayed in Figure 2 shows that even though the disk is stabilized against all small-scale disturbances and against axisymmetric disturbances according to Toomre's formula, it is still unstable against relatively slowly growing nonaxisymmetric disturbances. After two rotations the system has assumed a barshaped structure which changes very little during the following five rotations. At 


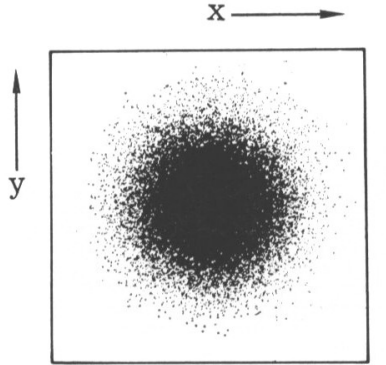

$\mathrm{t}=0$

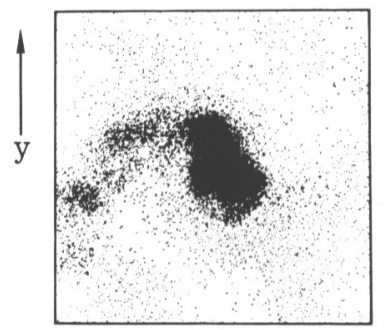

$\mathrm{t}=1.5$

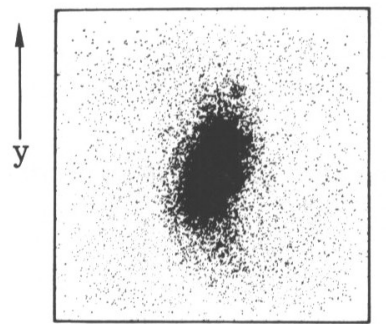

$\mathrm{t}=3.0$

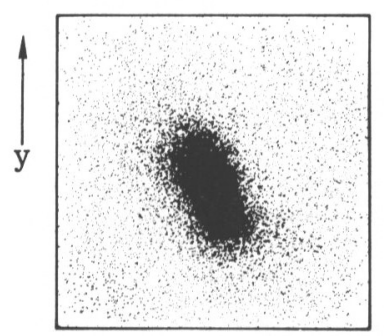

$t=5.0$

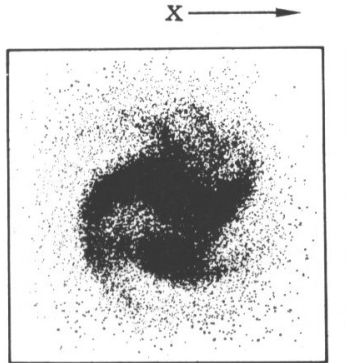

$t=0.5$

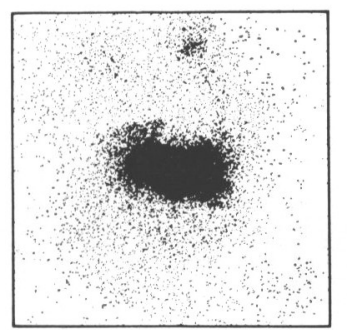

$\mathrm{t}=\mathbf{2 . 0}$

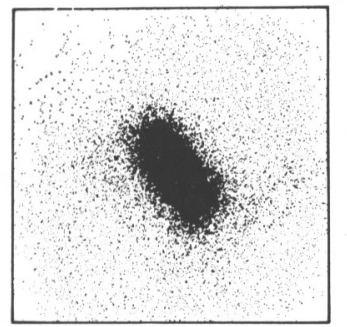

$t=3.5$

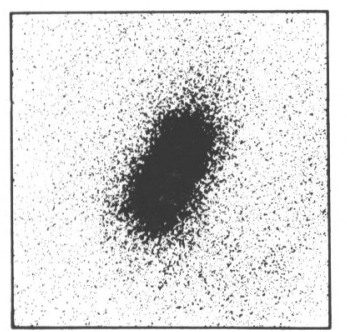

$\mathrm{t}=6.0$

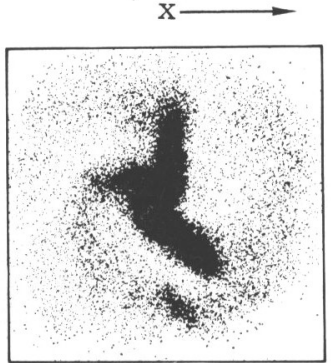

$\mathrm{t}=1.0$

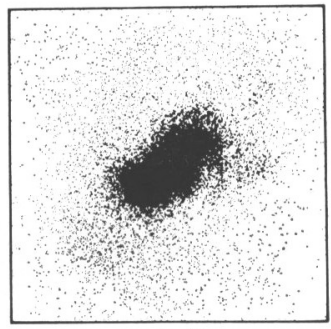

$t=2.5$

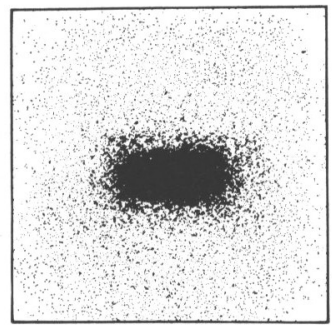

$\mathrm{t}=4.0$

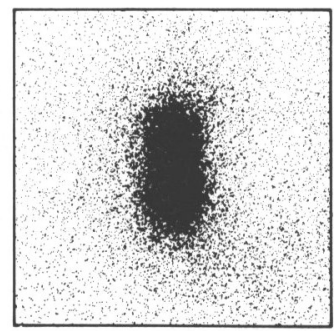

$t=7.5$

Fig. 2. Evolution of an initially stationary disk of stars with a Gaussian density distribution and with an initial velocity dispersion equal to $\sigma_{r, \min }$ and $\sigma_{\theta, \min }$ as calculated by Toomre.

$t=2.0$ the bar is rotating with a period of about 1.33 , at $t=3.5$ it rotates with a period of about 1.45. After $t=5.0$ the rotational period of the bar appears to remain constant at about 1.6. Note that these times are in units of $\tau_{0}$.

The change (error) in the total energy of the system is less than $1 \%$ for the first three rotations and the change in the angular momentum is about $0.1 \%$. It should 

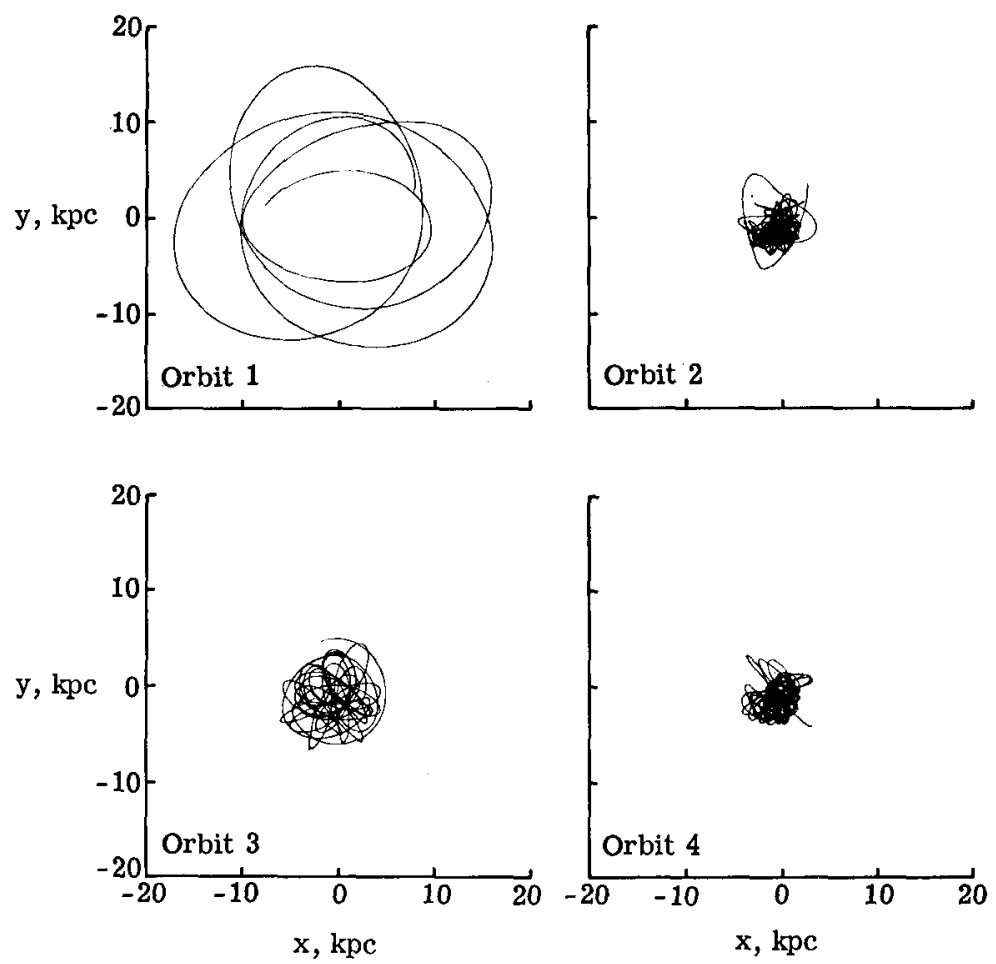

Fig. 3. Four individual star orbits for the disk with an initially Gaussian mass distribution.
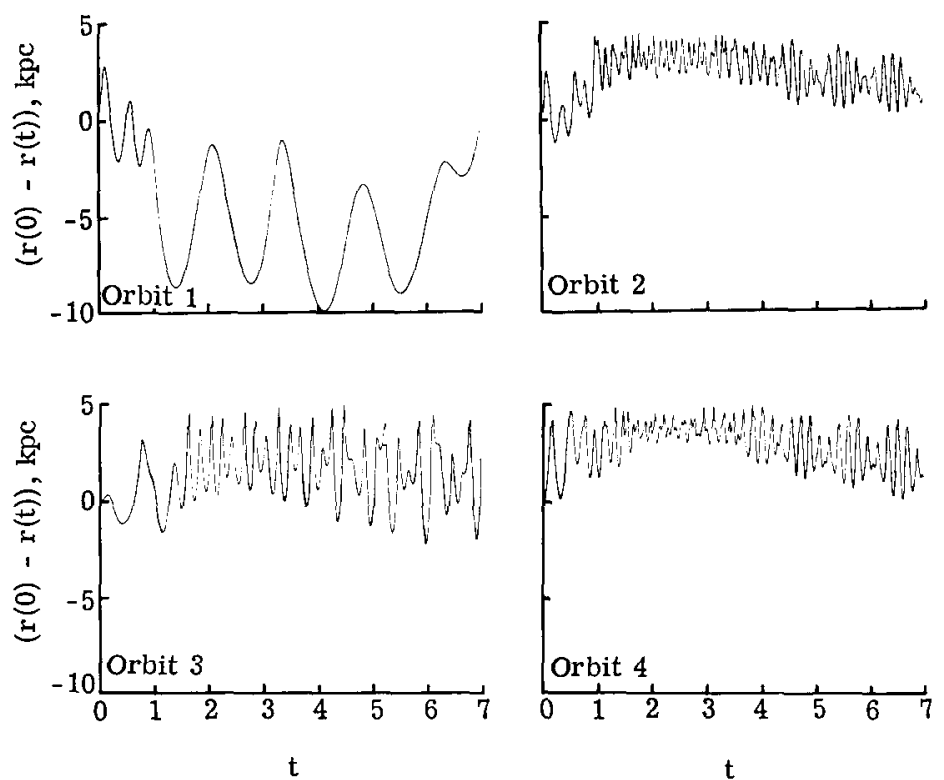

Fig. 4. Deviation from circular motion for the four star orbits shown in Figure 3. 

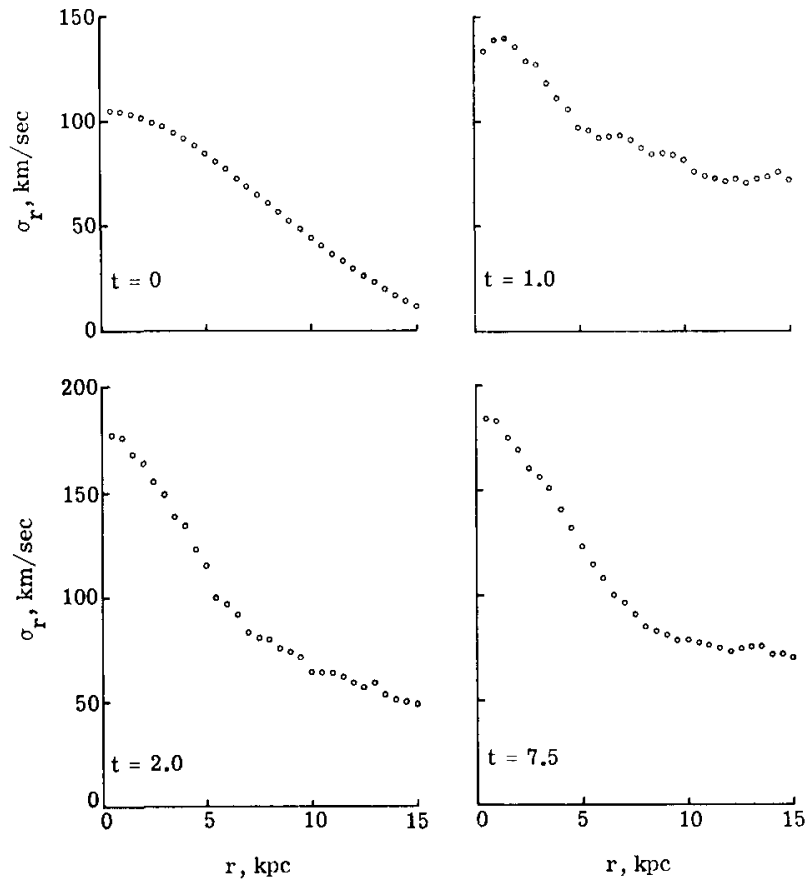

Fig. 5. Evolution of the radial velocity dispersion for the disk with a Gaussian mass distribution shown in Figure 2.
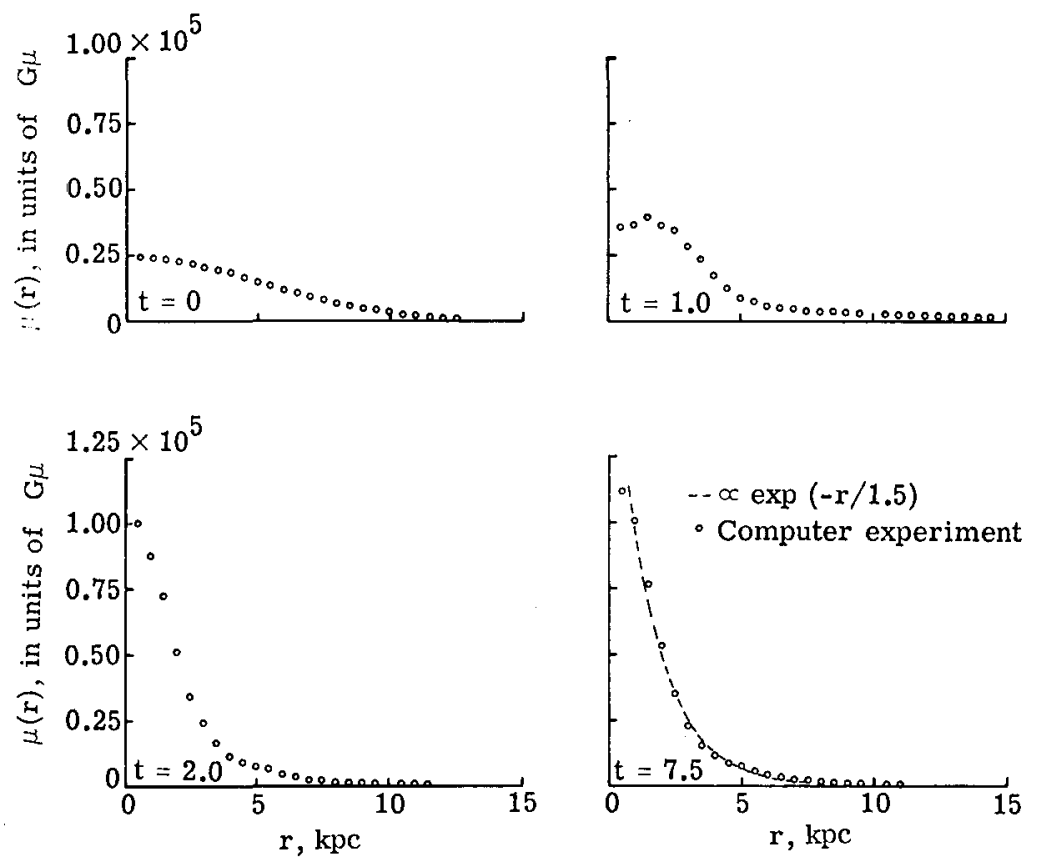

Fig. 6. Evolution of the mass density for the disk with a Gaussian mass distribution. The mass density is plotted in units of $\mathrm{G} \mu$ per $\mathrm{kpc}^{2}$. 
also be noted that at $t=0$ the virial theorem is satisfied; that is, the negative of the potential energy equals twice the kinetic energy of the disk.

Four individual star orbits taken at random from the 50000 stars in the disk are shown in Figure 3. The orbits are plotted by simply connecting the position of a star at each time step by straight line segments. The orbits indicate that stars initially near the center of the disk have a tendency to become trapped in even tighter orbits as the central mass density increases. Stars further out have a tendency to escape from the system. The orbits are plotted for the interval $t=0$ to $t=7$. The deviation of the star motion from circular orbits is displayed in Figure 4 which shows the difference between the initial star radius and the star radius at time $t$.

The frequency of the oscillations in the star orbits is that expected from epicyclic theory. In order to obtain more quantitative information than can be obtained from Figure 2, the disk is divided into a number of concentric rings each of $\frac{1}{2} \mathrm{kpc}$ width.

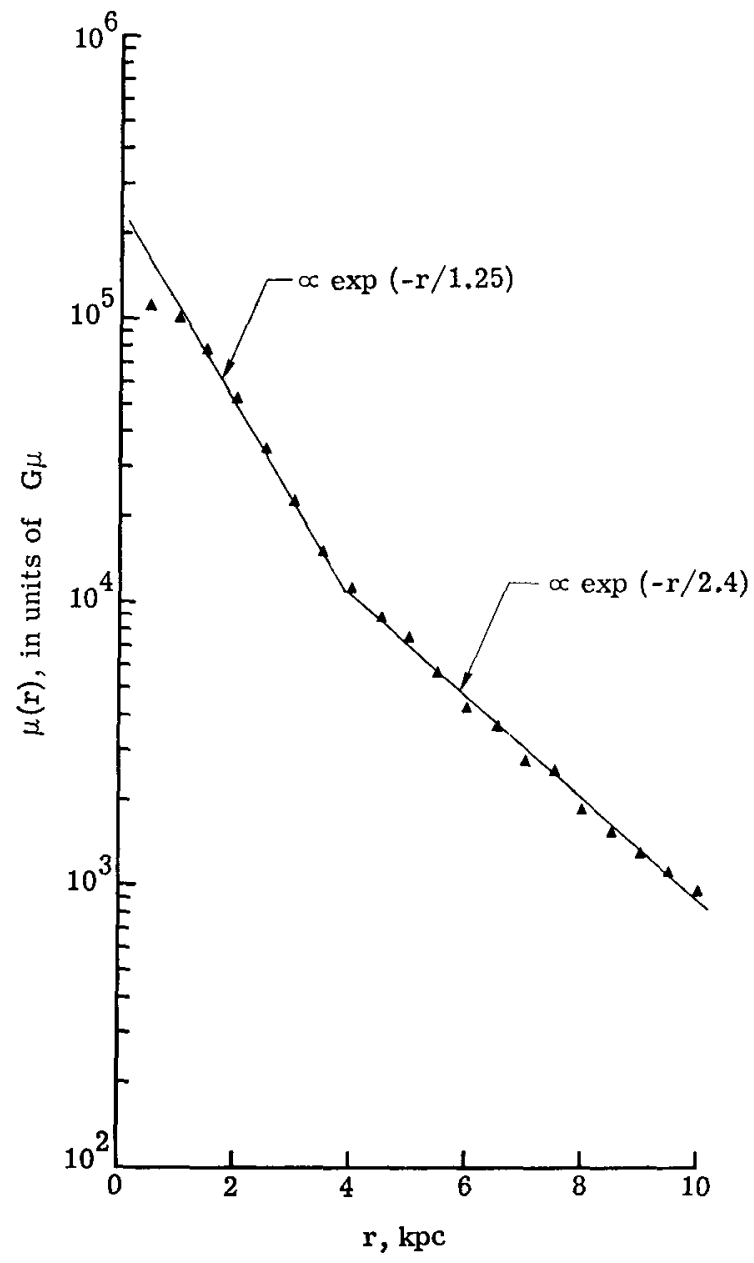

Fig. 7. Logarithmic plot of the density distribution at $t=7.5$ for the Gaussian disk. 
The radial dependence of various parameters averaged azimuthally over each ring is then obtained. Figure 5 shows the evolution of the radial velocity dispersion obtained in the above manner for the disk shown in Figure 2. Initially, the velocity dispersion for large $r$ increases rapidly as can be seen from a comparison of the results at $t=0$ and $t=0.25$. Again, the time shown is in units of $2 \pi / \omega_{0}$ at $r=10 \mathrm{kpc}$. After two rotations, the radial velocity dispersion in the central part of the disk has increased to about $175 \mathrm{~km} / \mathrm{s}$ from the initial value of $105 \mathrm{~km} / \mathrm{s}$. During the following five and one half rotations the velocity dispersion only increases slightly, indicating that at $t=2$ the system has nearly reached a steady state.

In interpreting the results shown in Figure 5 and in subsequent figures, the monaxisymmetric shape of the disk should be kept in mind. Figure 6 shows the evolution of the azimuthally averaged mass density as a function of radius. The figure shows that the central mass density of the disk increases by a factor of four during the first two rotations. Again there is little further change in the distribution during the following five and one half rotations. The dashed line shown at $t=7.5$ corresponds to an exponential density distribution with a scale length of $1.5 \mathrm{kpc}$,

$$
\mu(r) \propto \exp [-r / 1.5] \text {. }
$$

It can be seen that the exponential variation closely fits the mass distribution of the

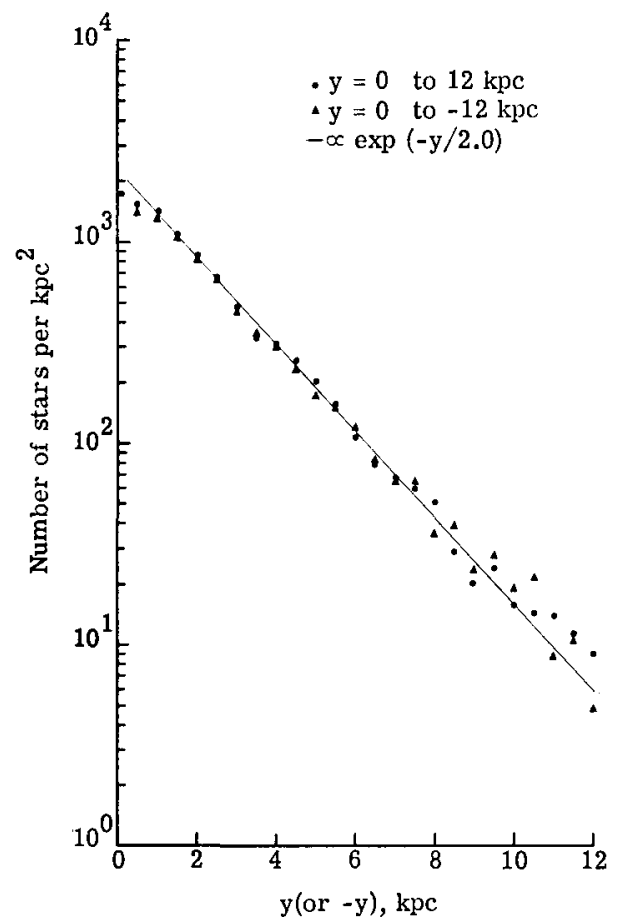

(a)

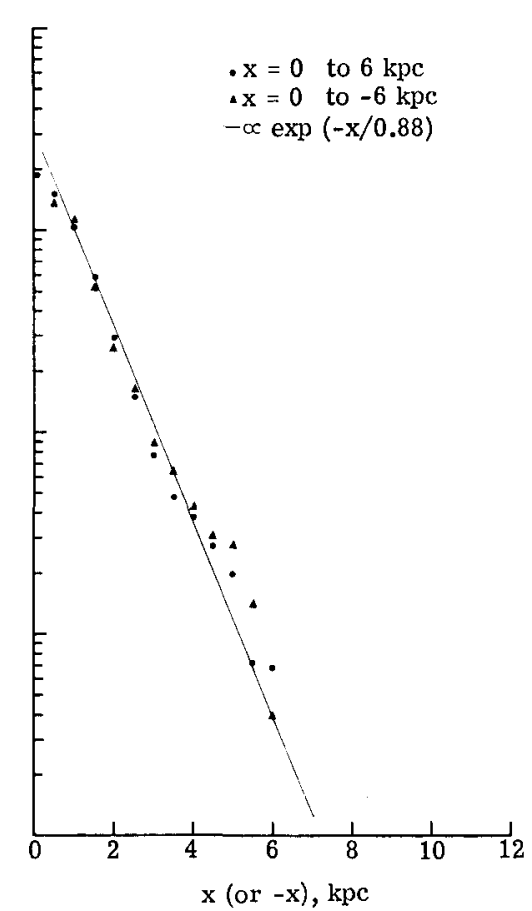

(b)

Fig. 8. Logarithmic plots of the density variation along the bar (a), and across the bar (b). 
disk. A better indication of how closely the mass density fits an exponential distribution can be obtained by plotting the azimuthally averaged density on a logarithmic scale. The result is shown in Figure 7 for $t=7.5$. Up to a radius of about four kpc the density varies as $\exp (-r / 1.25)$ and for $r$ larger than 4 it varies like $\exp (-r / 2.4)$. The variation shown in Figures 6 and 7 is the azimuthally averaged density variation and it is of interest to get an indication of the variation in any particular radial direction. At $t=7.5$ the bar is in a vertical position and from the number of stars in the cells along the $y$-direction for $x=0$ and along the $x$-direction for $y=0$ we obtain the variation along and across the bar. Note that $x=y=0$ represents the center of the system. The results are shown in Figure 8 where the number of stars per $\mathrm{kpc}^{2}$ is plotted on a logarithmic scale along the bar (a), and across the bar (b). Along the bar the variation is closely approximated by an exponential variation exp $(-y / 2.0)$ and across the bar it approximates $\exp (-x / 0.88)$. The variation shown in Figure 7 appears to be a combination of the exponential variation across and along the bar.

\section{Exponential Mass Distribution}

\section{A. INITIAL CONDITIONS}

For the disk with an initial Gaussian mass distribution it was found that the final mass density was closely described by an exponential distribution. It is therefore of interest to investigate whether a disk with an initially exponential mass distribution will remain stable. The initial mass density for a disk given by

$$
\mu(r)=\mu(0) \exp \left(-r / r_{0}\right)
$$

where $\sigma(0)$ is the central density and $r_{0}=3 \mathrm{kpc}$ was investigated. The initial values of $\omega_{0}$ and $\omega$ as a function of $r$ are obtained by finite difference methods from the computer model. The disk contains 50000 stars. The mass per star is $G m=60$ and the initial mass density was cut off at $15 \mathrm{kpc}$ (or five-scale lengths). This cutoff should not effect the dynamics of the disk since only $4 \%$ of the total mass for the exponential distribution lies outside $15 \mathrm{kpc}$. The initial velocity dispersion was given by Equations (4) and (5). It was found (Hohl, 1970) for the exponential disk that the evolution is note quite as violent as was the case for the Gaussian mass distribution. However, after only 1.5 rotations the system had again assumed a bar-shaped structure with a halo of stars moving in larger orbits around the central bar. Only slow changes in the shape of the system occurred after $t=1.5$.

In an attempt to force a more stable disk the disk with the initial exponential distribution just described was evolved up to five rotations with the constraint that every quarter rotation the stars were uniformly redistributed in the azimuthal direction. This was done by using a pseudo random number generator. The radial and azimuthal velocity components of the stars remained unchanged. The primary effect was an approximately $15 \%$ increase in the velocity dispersion and an about $20 \%$ increase in the central density. The evolution of the disk starting from this slightly 
hotter and more centrally condensed exponential disk is discussed in the next section.

B. EVOLUTION OF THE DISK

Figure 9 displays the evolution of the exponential disk. The evolution is not quite as violent as was the case for the Gaussian mass distribution. In addition the final
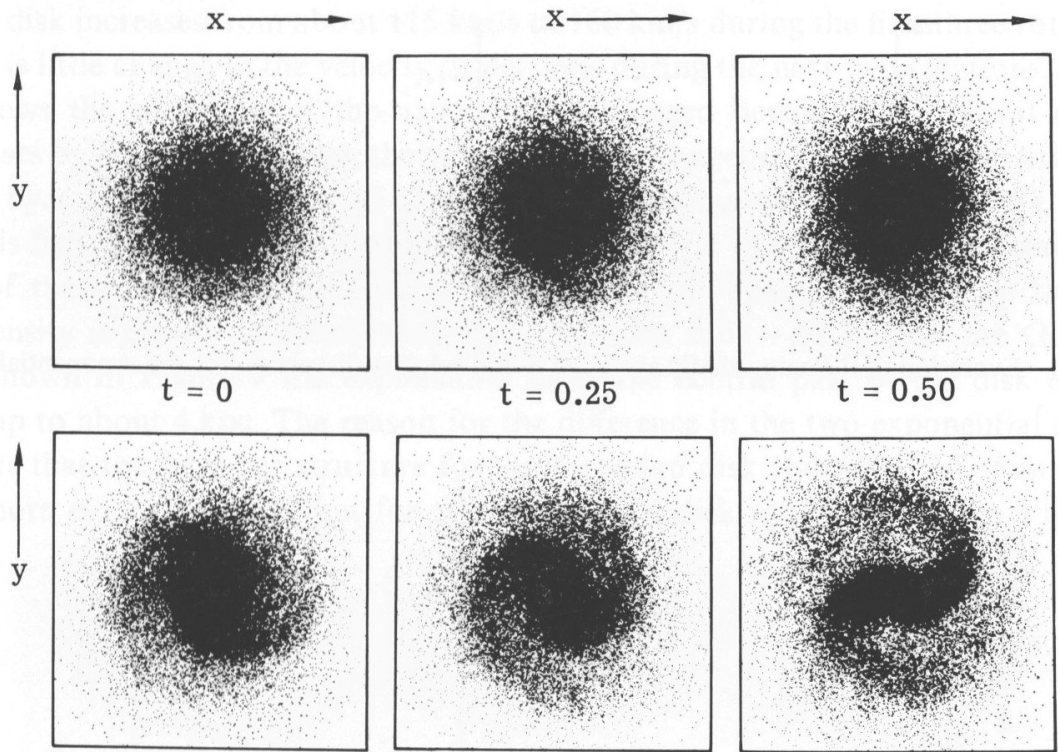

$\mathrm{t}=0.75$

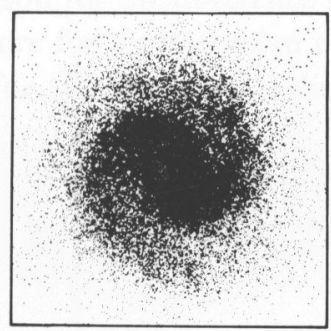

$\mathrm{t}=1.00$
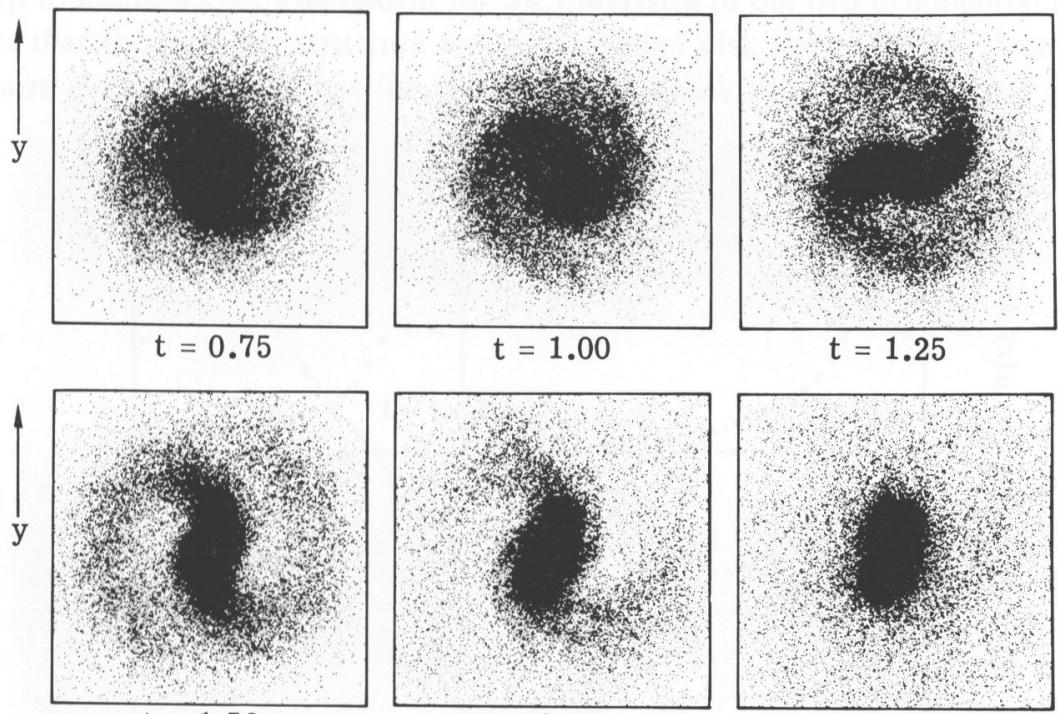

$\mathrm{t}=2.00$
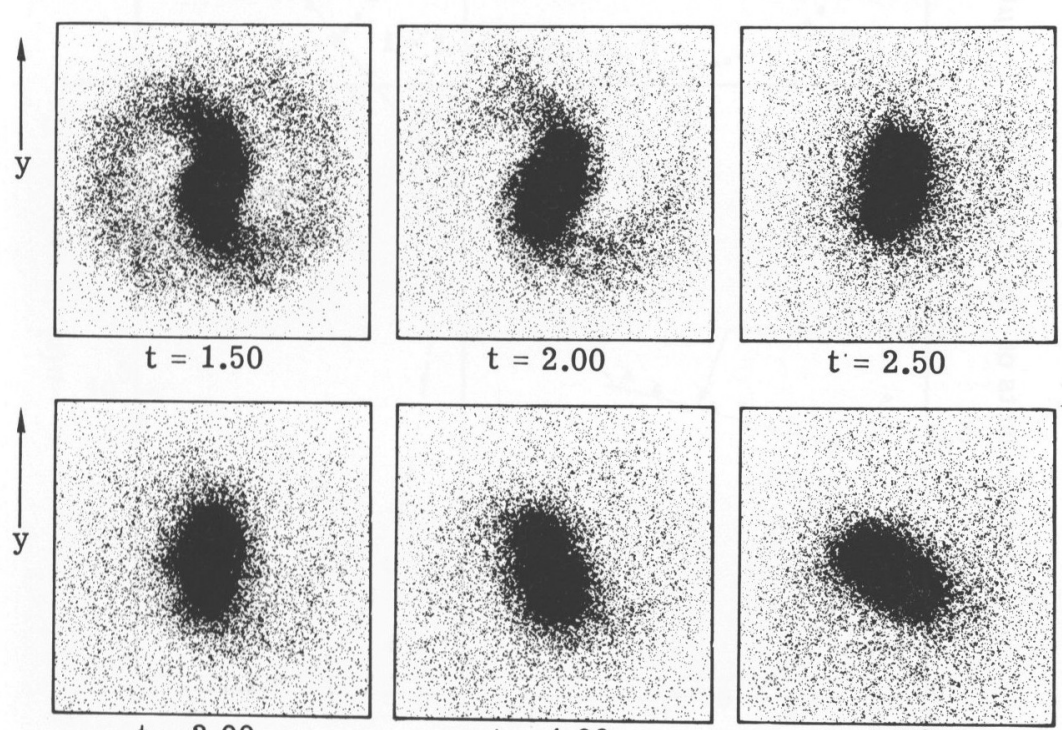

$\mathrm{t}=4.00$

$t^{\prime}=2.50$

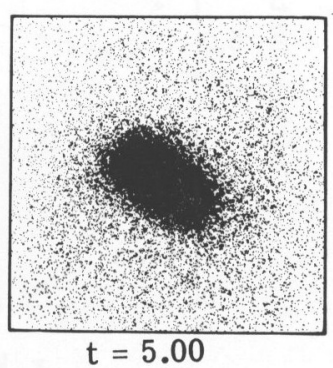

Fig. 9. Evolution of a disk of stars with an initially exponential mass distribution. 

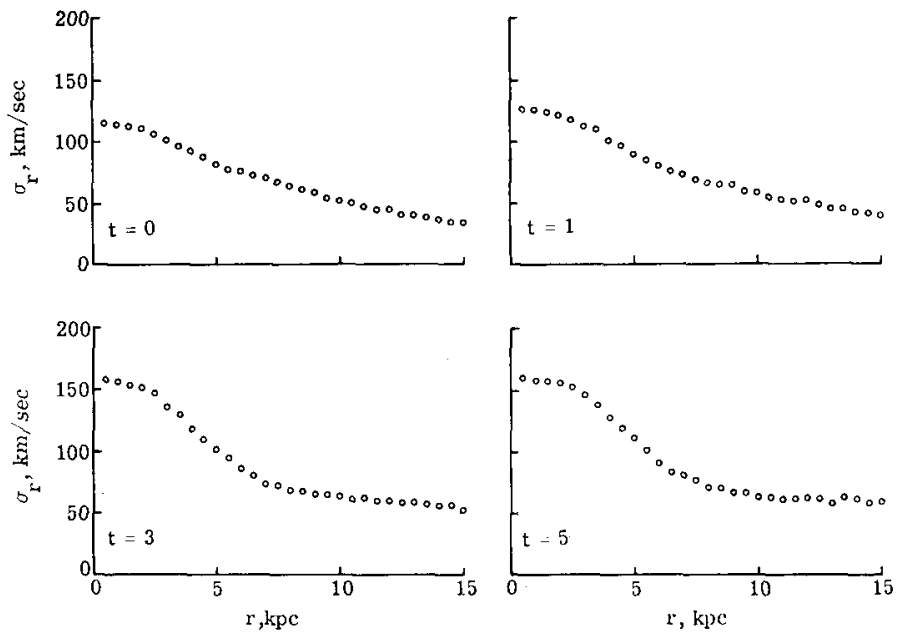

Fig. 10. Evolution of the azimuthally averaged radial velocity dispersion for the exponential disk.
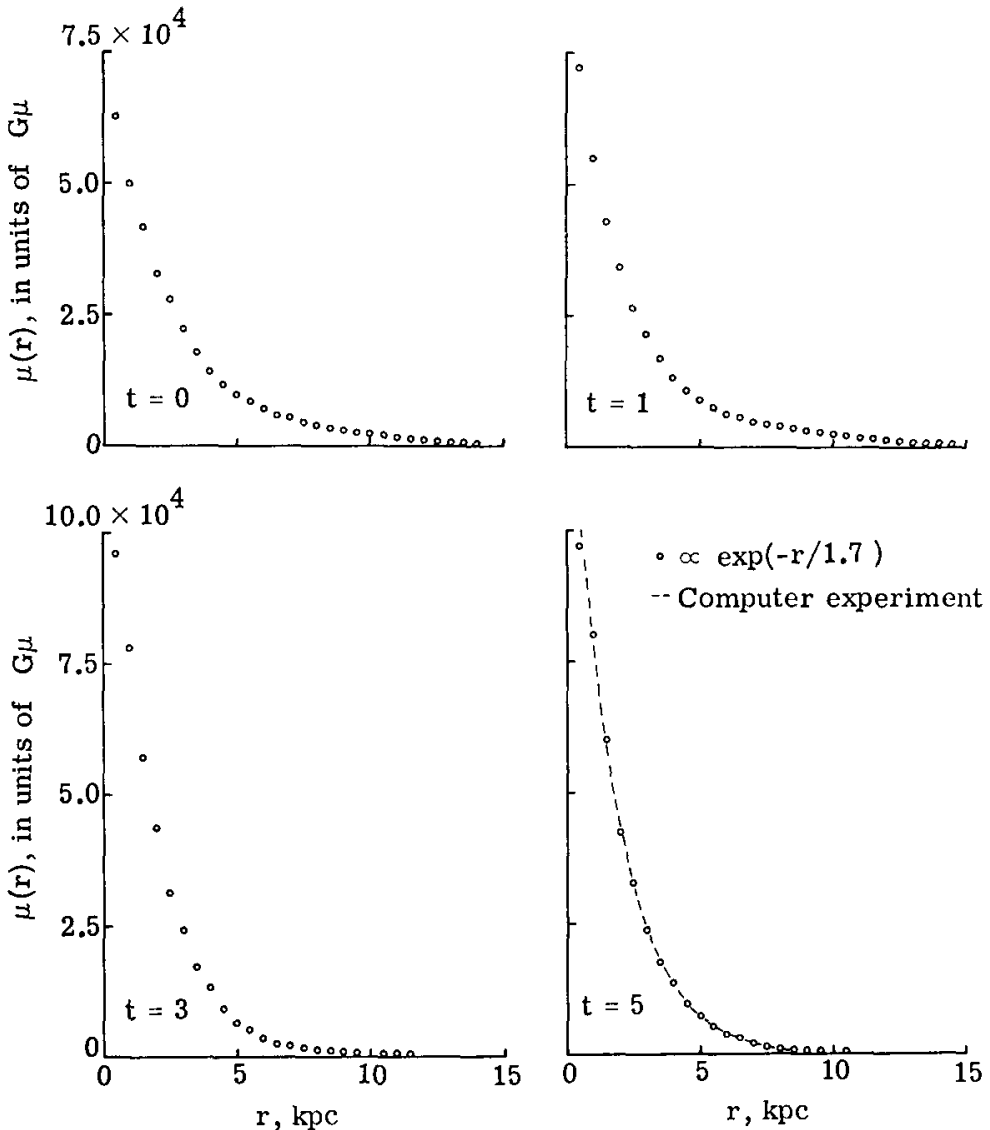

Fig. 11. Evolution of the azimuthally averaged density for the exponential disk. 
bar structure is shorter and 'fatter' than that shown in Figure 2. Even though the 'initial' velocity dispersion is about $15 \%$ larger than that calculated by Toomre for the suppression of axisymmetric instability, the disk quickly assumes a bar-shaped structure, in less than two rotations. Note that the time shown is in rotational periods at $r=10 \mathrm{kpc}$ and that the rectangle enclosing the disk is at $y$ or $x= \pm 19 \mathrm{kpc}$.

The evolution of the azimuthally averaged radial velocity dispersion for the exponential disk is shown in Figure 10. The velocity dispersion in the central region of the disk increases from about $115 \mathrm{~km} / \mathrm{s}$ to $160 \mathrm{~km} / \mathrm{s}$ during the first three rotations. There is little change in the velocity dispersions during the next two rotations. Figure 11 shows the evolution of the azimuthally averaged density. The central density increases by about $50 \%$ during the evolution and changes from an initial distribution given by $\exp (-r / 3)$ to a final distribution approximated by $\exp (-r / 1.7)$. Again there is little change in the distribution during the last two rotations. A logarithmic plot of the density distribution at $t=5$ is shown in Figure 12 . For $r$ up to $8 \mathrm{kpc}$ the density is closely approximated by a variation $\exp (-r / 1.7)$. For the Gaussian case shown in Figure 7 the exponential fit in the central part of the disk extends only up to about $4 \mathrm{kpc}$. The reason for the difference in the two exponential density plots is that the final bar struzture for the Gaussian disk shown at $t=7.5$ in Figure 2 is more elongated than that for the exponential disk shown at $t=5$ in Figure 9.

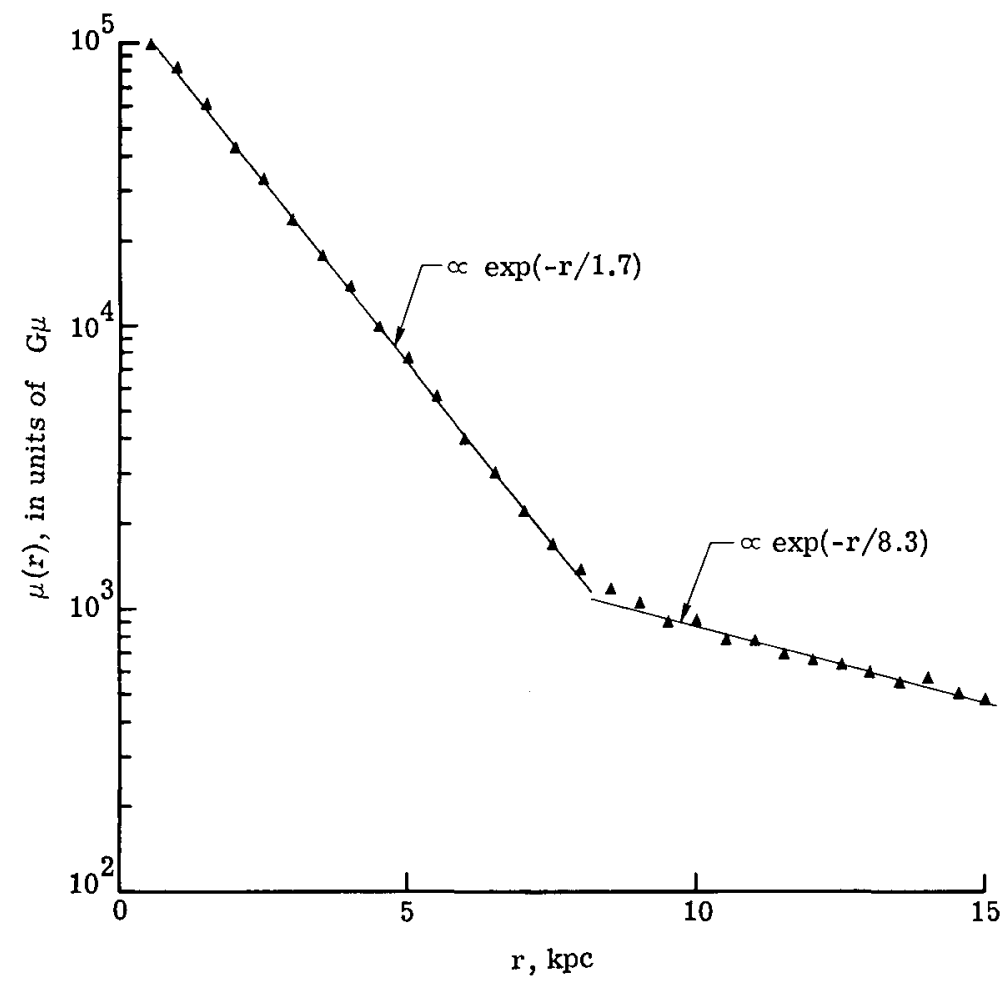

Fig. 12. Logarithmic plot of the density distribution at $t=5$ for the exponential disk. 


\section{Stable Axisymmetric Disk}

Disks of stars with initial conditions generated according to analytical expressions such as Equations (3) to (6) were found to be unstable and to finally assume a steady state with a central bar-shaped structure. The evolution of the warm disks is found to be very similar irrespective of whether the initial mass distribution is Gaussian, exponential or some other distribution (Hohl, 1970). Also, the stationary state finally reached by the disk results in a rather hot population of stars in the outer portions of the disk. In order to generate an axisymmetric stable disk the final distribution of stars of an evolved disk like that shown at $t=5$ in Figure 9 was used as an initial condition after symmetrizing out the bar structure. All stars keep the same velocity components and radius, except that they are now axisymmetrically distributed by means of a random number generator. The evolution of such a disk is shown in Figure 13 for six rotations. The rectangular border surrounding the disk is at $x= \pm 32$ $\mathrm{kpc}$ and $y= \pm 32 \mathrm{kpc}$. Also, the disk contains 100000 stars each of mass $G m=30$. The disk is stable and all parameters remain constant during the evolution. For
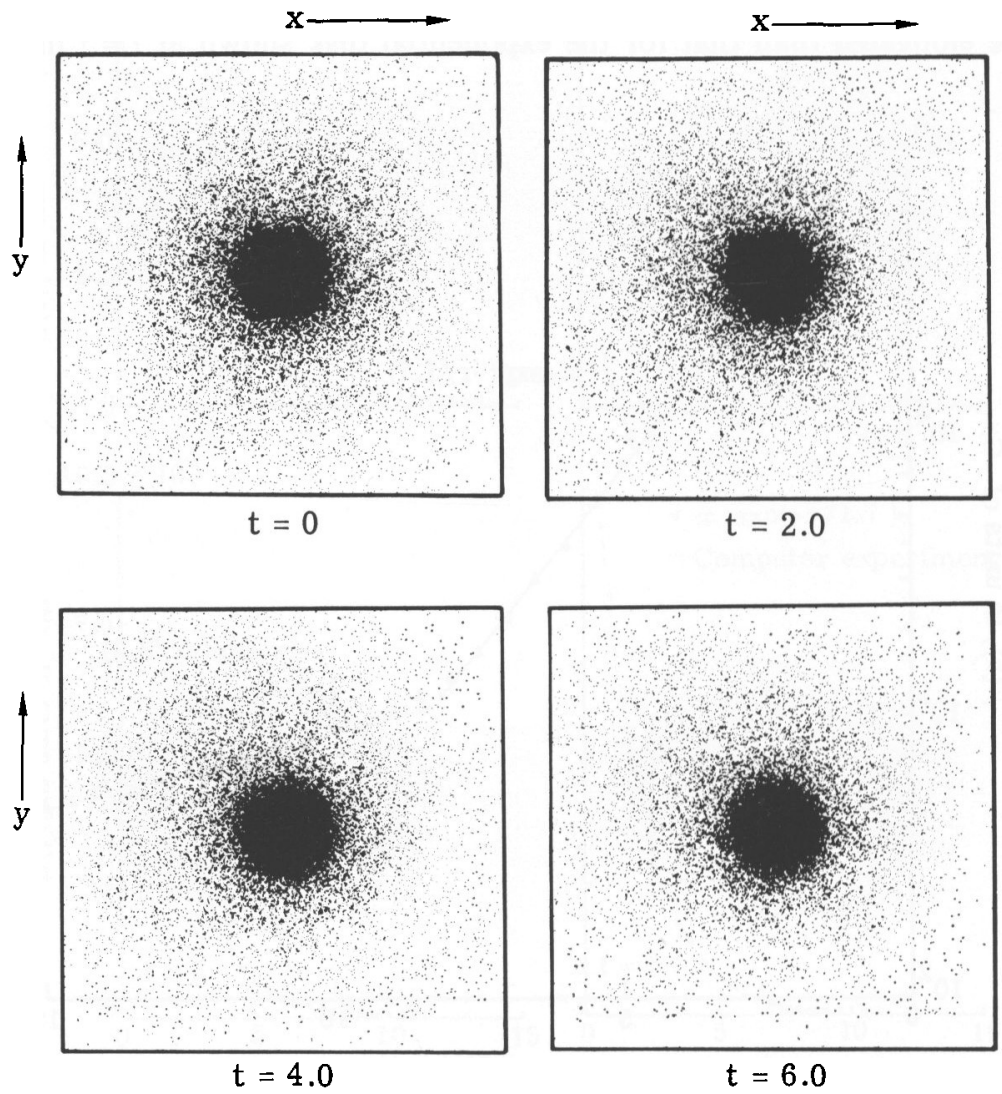

Fig. 13. Evolution of a stable axisymmetric disk of 100000 stars. 


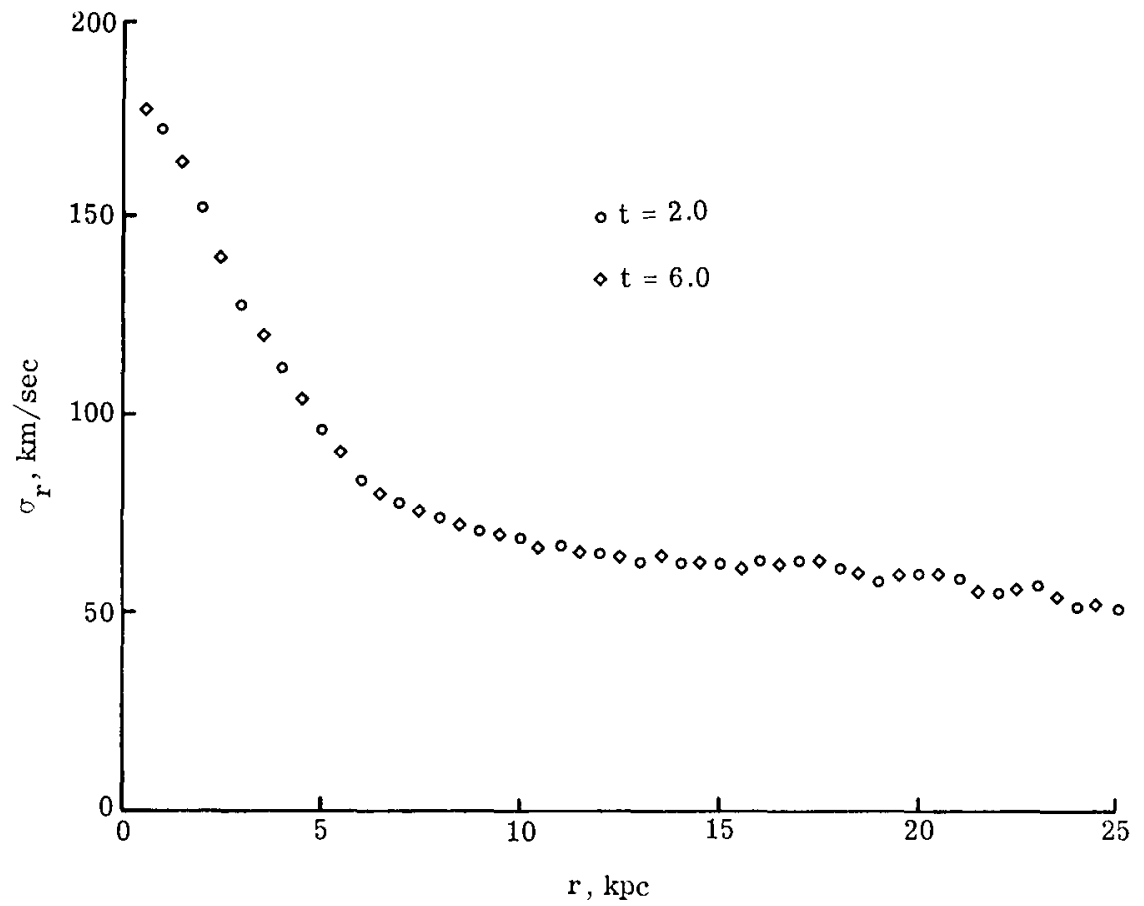

Fig. 14. Variation of the radial velocity dispersion for the stable disk shown in Figure 13.

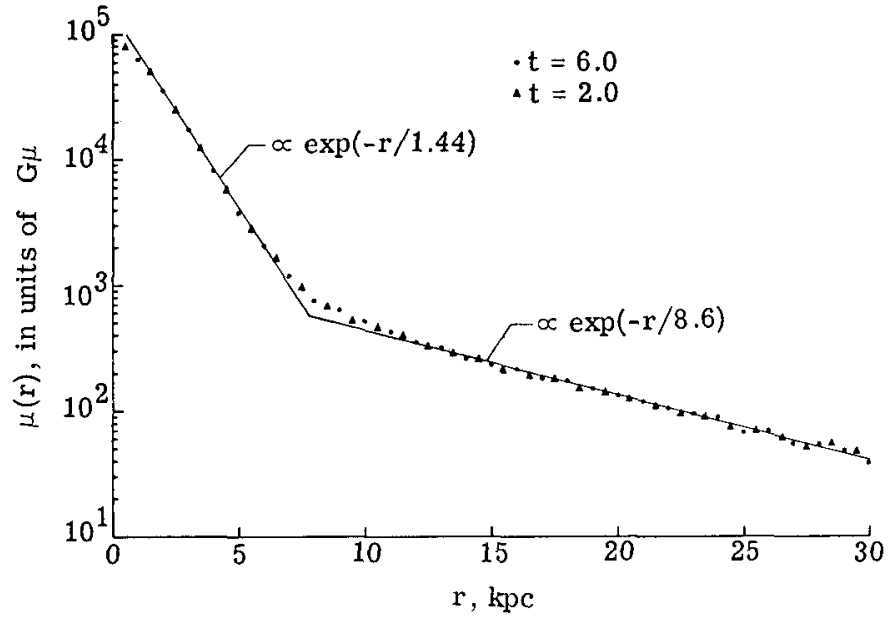

Fig. 15. Variation of the density for the stable disk shown in Figure 13. 
example, the radial velocity dispersion shown in Figure 14 shows no change for the results after 2 and 6 rotations. A similar result is shown in Figure 15 for the density variation. Again the variation of the density is closely described by an exponential variation over most of the disk.

\section{Gravitational Two-Stream Instability}

The gravitational two-stream instability was investigated for 100000 star systems with a given initial velocity dispersion. A pseudo random number generator was used to obtain a nearly uniform initial distribution of stars. A uniform distribution of one half of the stars (50000 stars) has superposed on the initial velocity dispersion, $\sigma$, a constant streaming velocity, $V$, in the positive $x$-direction. The remaining half of the stars has superposed a streaming velocity, $V$, in the negative $x$-direction. Figure 16 shows the results for four different values of the ratio of streaming velocity to velocity dispersion, $V / \sigma$. Since the model is now doubly periodic, a star leaving the rectangular region at one side will enter the region again from the opposite side with the same velocity components. The mass per star is $G m=0.5$ and the density per cell ( 64 by 64 array of cells) is $G \mu=12.5$. Times shown in Figure 16 are in units of $\tau_{0}=1 / \sqrt{G \mu}$. Figure 16(a) shows the Jean's instability for zero streaming, $V / \sigma=0$. Toomre (1964) has given the Jeans' length for this plane stellary system as

$$
\lambda_{J}=\frac{\sigma^{2}}{G \mu} .
$$

For the present system $\sigma$ is 5 ( 5 cell dimensions per $\tau_{0}$ ). The measured diameter of the condensations shown in Figure $16(\mathrm{a})$ is $2 \lambda_{J}$, indicating that disturbances with wavelength $2 \lambda_{J}$ have the fastest growth rate. Figure $16(\mathrm{~b})$ and (c) show that the dynamics of the two-stream instability changes very little up to streaming velocities of $V / \sigma=1.0$. For streaming velocities greater than $V / \sigma=1.0$, Figure $16(\mathrm{~d})$ shows that there is a marked change in the evolution and final structure of the instability. The system now displays an elongated filamentary structure similar to that found in certain spiral galaxies.

\section{Concluding Remarks}

Numerical experiments with a computer model for isolated disk galaxies have been performed for a large number of initial conditions. The results for three cases of initially balanced disks of stars were presented. By adding a sufficient initial velocity dispersion (as given by Toomre, for example) all fast-growing small-scale disturbances could be stabilized. However, all such 'stabilized' disks investigated were found to be unstable against more slowly growing long wave-length disturbances and in less than two rotations the disks assumed a bar-shaped or elliptical structure. Various modifications to the model were tried in an attempt to generate a stable axisymmetric disk. For example, a fraction of the mass in the central portion of the disk was held 


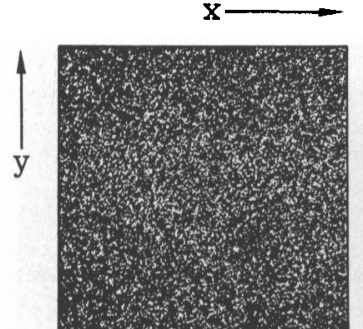

$\mathrm{t}=0$

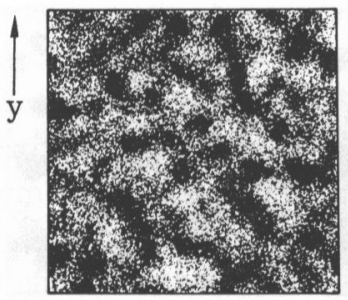

$\mathrm{t}=2.4$

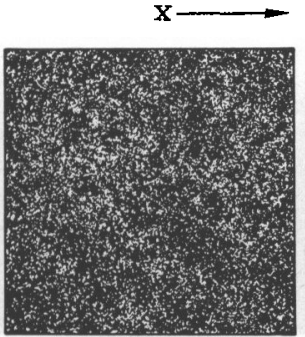

$\mathrm{t}=0.8$

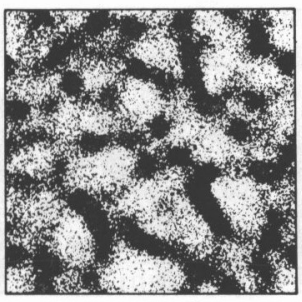

$\mathrm{t}=3.2$

(a) $\mathrm{V} / \sigma=0$.

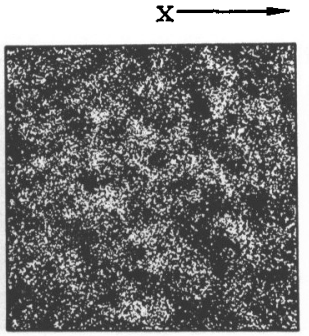

$\mathrm{t}=1.6$

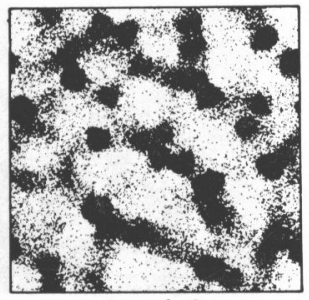

$\mathrm{t}=4.0$

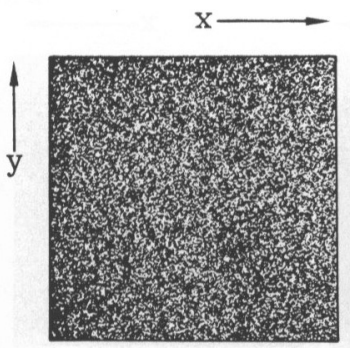

$\mathrm{t}=0$

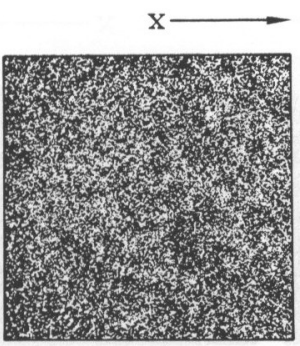

$\mathrm{t}=0.8$

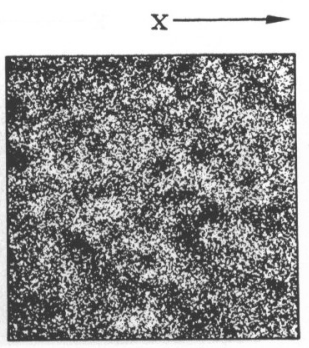

$t=1.6$

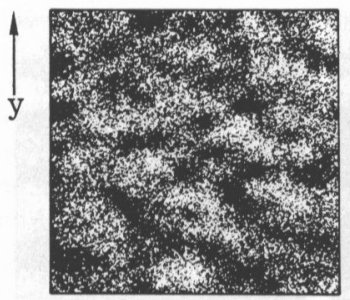

$\mathrm{t}=2.4$

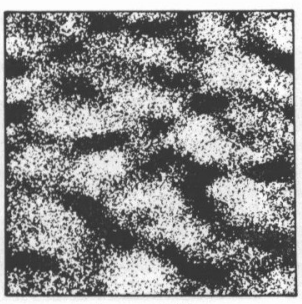

$\mathrm{t}=3.2$

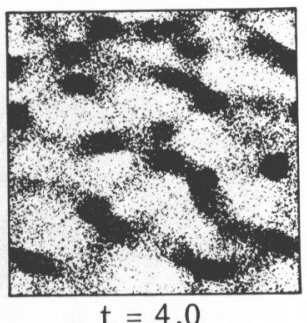

(b) $\mathrm{V} / \sigma=0.5$.

Fig. 16a-b. Evolution of the two-stream instability for four different streaming velocity (a) $V / \sigma=0$, (b) $V / \sigma=0.5$, (c) $V / \sigma=1.0$, and $V / \sigma=2.0$. 
FRANK HOHL

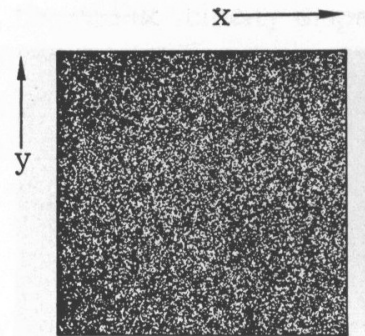

$t=0$

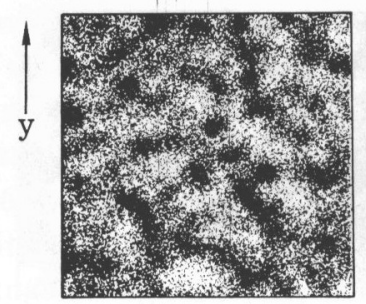

$\mathrm{t}=2.4$

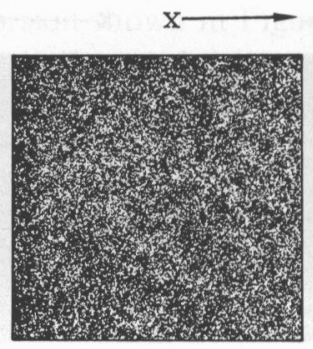

$\mathrm{t}=0.8$

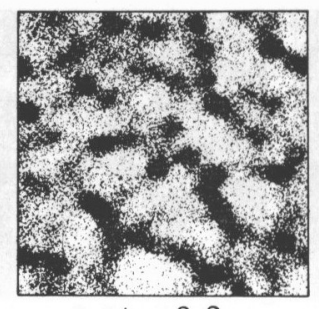

$\mathrm{t}=3.2$

(c) $\mathrm{V} / \sigma=1.0$.

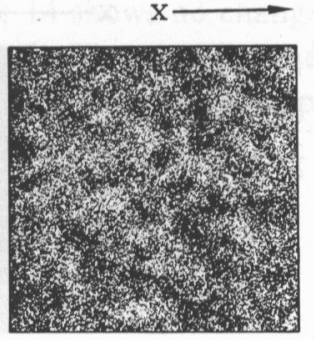

$\mathrm{t}=1.6$

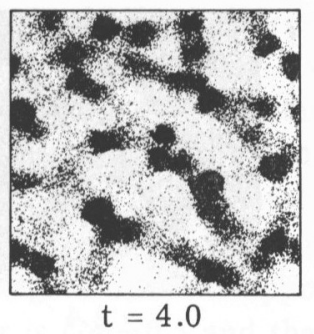

$\mathrm{t}=4.0$

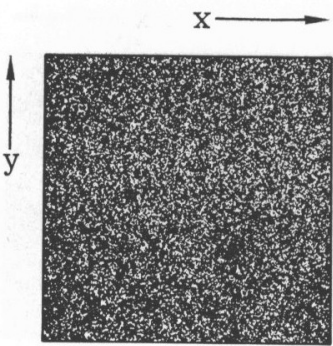

$\mathrm{t}=0$

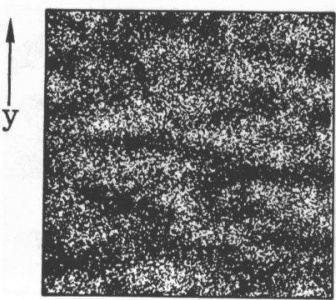

$\mathrm{t}=2.4$
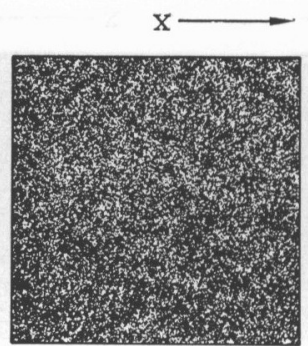

$t=0.8$

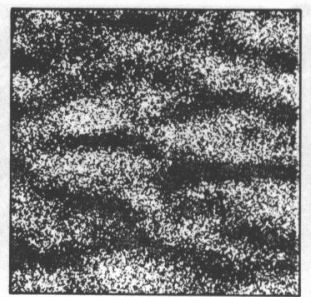

$\mathrm{t}=3.2$

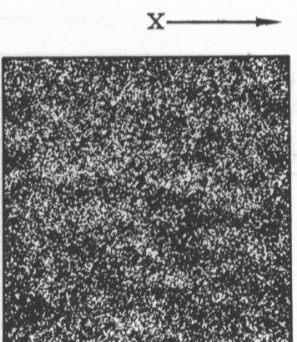

$t=1.6$

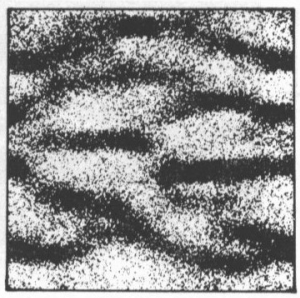

$\mathrm{t}=4.0$

(d) $\mathrm{V} / \sigma=2.0$.

Fig. 16c-d. 
fixed. Of course, if the fraction of mass held fixed becomes large (say 70 or $80 \%$ ) then axisymmetric disks can easily be obtained. The final velocity dispersion for the disks is generally found to be from 100 to $200 \%$ larger than $\sigma_{r \text {, min }}$ as calculated by Toomre. By evolving a disk of stars on the computer until it reached a steady state and by then symmetrizing out the central bar structure it was possible to generate a stable axisymmetric disk.

An interesting result of the calculations is that for the disks of stars so far investigated the final mass distribution in the radial direction can be closely approximated by an exponential variation irrespective of the initial conditions. This result is in agreement with observational evidence (Freeman, 1970) which indicates that the luminosity distribution of many $\mathrm{SO}$ and spiral galaxies can be approximated by an exponential distribution.

In investigating the gravitational instability it was found that the condensations which form have dimensions equal to about two Jeans lengths. Increasing the streaming velocity from zero to a value equal to the velocity dispersion of the system had little effect on the dynamics of the instability. For streaming velocities larger than the velocity dispersion, the system developed elongated filamentary structure.

\section{Acknowledgement}

I wish to thank Professor Alar Toomre for his many suggestions and contributions to this work.

\section{References}

Chandrasekhar, S.: 1960, Principles of Stellar Dynamics, Dover Publ., Inc., New York.

Cuperman, S., Goldstein, S., and Lecar, M.: 1969, Monthly Notices Roy. Astron. Soc. 146, 161.

Freeman, K. C.: 1970, Astrophys. J. 160, 811.

Hénon, M.: 1964, Ann. Astrophys. 27, 83.

Hockney, R. W.: 1967, Astrophys. J. 150, 797.

Hockney, R. W. and Hohl, F.: 1969, Astron. J. 74, 1102.

Hohl, F.: 1968a, NASA TR R-289.

Hohl, F.: 1968b, Bull. Astron. 3, 227.

Hohl, F.: 1969, NASA TN D-5200.

Hohl, F.: 1970a, in W. Becker and G. Contopoulos (eds.), 'The Spiral Structure of our Galaxy', IAU Symp. 38, 368.

Hohl, F.: 1970b, NASA TR R-343.

Hohl, F. and Campbell, J. W.: 1968, Astron. J. 73, 611.

Hohl, F. and Campbell, J. W.: 1969, NASA TN D-5540.

Hohl, F. and Feix, M. R.: 1967, Astrophys. J. 147, 1164.

Hohl, F. and Hockney, R. W.: 1969, J. Comput. Phys. 4, 306.

Lecar, M.: 1966, in G. Contopoulos (ed.), 'The Theory of Orbits in the Solar System and in Stellar Systems', IAU Symp. 25, 46.

Miller, R. H. and Prendergast, K. H.: 1968, Astrophys. J. 151, 699.

Miller, R. H., Prendergast, K. H., and Quirk, W. J.: 1970, 'Numerical Experiments on Spiral Structure', to be published.

Toomre, A.: 1963, Astrophys. J. 138, 385.

Toomre, A.: 1964, Astrophys. J. 139, 1217. 